\title{
Fibroelastosis endocárdica: aspectos clínicos y ecocardiográfico
}

\author{
Dris. Odette Farrú A.: Dra. Patricia Llrriola B.; Dr. Antonio Solís A.1 \\ Endocardial fibroelastosis
}

\begin{abstract}
The clinical findings in seventeen chiksen with primary endocardial fibroelastosis are analyst. Ten patients had r'puted echocardiographic studies $(M-m o d e)$ and in cight the diagnosis was confirmed at necropsy. In addition to the classical signs of congestive tnyocardiopathy, a definite thickening of the endocardium and tnitral subvalualar apparatus wa observed in the echocardiographic examination. In one wase, the thickening progressively increased till death, and his histological abnormalitics suggested that the disease may envolve in bouts with progressive endocardial fibroclastik thickening. 'I'leere was an overall mortality sate of $47 \%$ but we believe that the survival depends mostly on precocity, intensity and persistence of treatment. Thi use of vasodikators drugs seems to improve prognosis and survival.

(Key words: Endocardial ibrotlastonjs. Myocardiopathy, contestive. Congestive heate failute. Endocardial thickening. Fichocard jography).
\end{abstract}

La libroelastosis endocárdica (FEE) es una afección particular dil lactante menor muy controvertidit, especialtuente en cuanto it su pronóstico: según algunos. la evolución es fatal en la casi totalidad de los casos ${ }^{2}$. según otros, en sólo cerca de la mitad ${ }^{3,4}$.

Los signos ecocardingráfieos conocidos de la FEF son aquellos inespecíficos de una miocardiopatiat congestiva ${ }^{5}$. Dada la dificultad clinica para demostrar con certeza la existenciá de una FEE, seria deseable contar con un metodo iticruento que pernita asegurar el diagnóstico.

FI objeto del presence trabajo es, por und parte, estudiar en nuestro medio las caracteristicas clínico evolutivals y los posibles factores que influyen en el pronóstico de la cnfermedad $y$ por otra, describir ciertos signos ecocardiogríficos que cremos específicos de este tipo de miocardiopat ía congestiva.

\section{MATERLAI. Y METODO}

Se estudian 17 casoe de l-1: aislada diagnosticadas clínicamente en los últimos 8 arios $y$ en los tuales se contó con un seztimiento adecuado. I: 8 , el diagnistito se confirmó en la autopsia. Todos los pacientes tenían estudio clinico, radiológico y electuocadiográ fico compleio. 10 estudio scocardiogrático (Modo- M) y uno estudio hemodinámico.

1. Inidad de Cardiología, l'epartansento de Podjatria, llospital Robetto del Río.

\section{RESULTADOS}

Doce pacientes eratl de sexo femenino, (relación $F: M=2,4: 1$ ). La edad promedio de comienzo de los sintonas fue de 4 meses, (rango 14 días y 18 meses): 10 niños consultaron antes de $\operatorname{los} 2$ meses de edad, sólo uno después dol año. Todos lenian insuficiencia cardiaca congestiva grave, de comien\%o brusco, de dificil manejo, sin soplos significativos. En la mayoría de los casos ${ }^{14}$, e] coniemzo de los sintontas cardíacos estuvo precedido pocos días antes por fiebre y molestias sugerentes de infección de las vias respiratorias altas, aparentemente viral. Radiológicamente todos los pacientes tenían cardiomegalia global significativa, (Fig. 1) con Jatidos de amplitud disninujda en la radioscopía. E] electrocurdiograma (ECG) (Fig. 2) mostraba, en todos, marcados signos de reacción c hiperirofia ventriculat izquierda (VI) con ondas $R$ de muy alto voltaje en las derivaciones precordiales izquierdas y profundas alteraciones de la repolarización ventricular: ondas $T$ invertidas en 11 casos y planas on V5 y V6 en otros 6 pacientes.

Se realizaron estudios virológicos en 4 pacientes, sjendo positivos en 3 , para virus Coxsackie y negativo en el cuasto.

En 10 casos se realizó estudio ecocardiográ⿴j. co Modo-M, en 8 seriado, comprobándose marcada dilatación del ventrículo y la auricula izquierdos, excursión reducida del septum y de la 


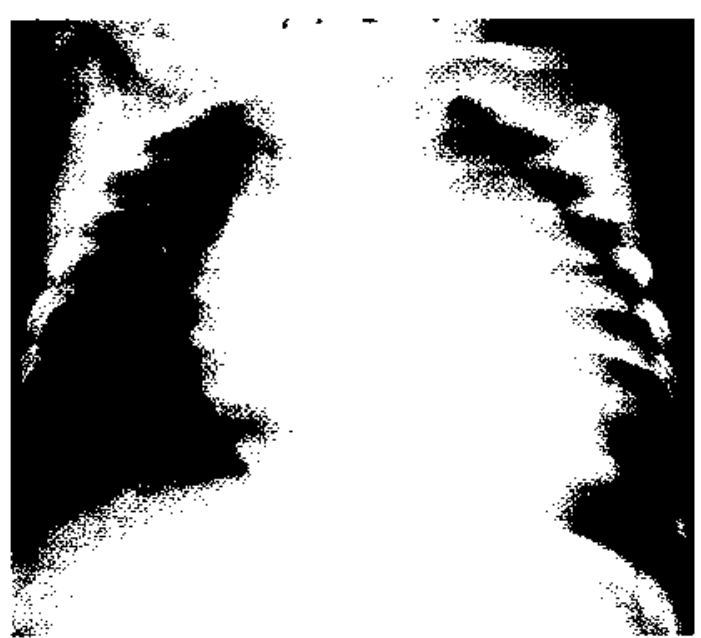

Figura 1: Fibroclastosis endocárdica. Telesadiografía de totax frontal: cardiomegalia global, circulación putnonat norsal.
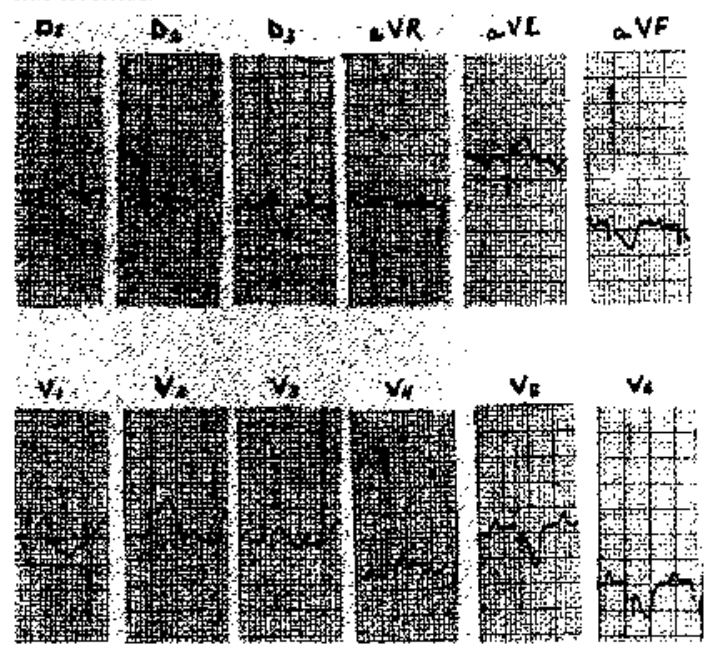

Figura 2: Fibroelastosis endocárdica. ( 3 meses de edad) Electrocardiograma. Gran reacción ventricular izquicrda. Ondas $R$ muy altas in $\mathrm{L}_{2}, \mathrm{D}_{3}$, aVF y $\mathrm{V}_{6}$

pared posterior del VI, fracción de acortamiento de éste muy disninuida (entre 10\% y $19 \%$. promedio $15 \%$ ), inlervalos sistólicos de V1 muy alterados (PPE/PE muy prolongado). excursión
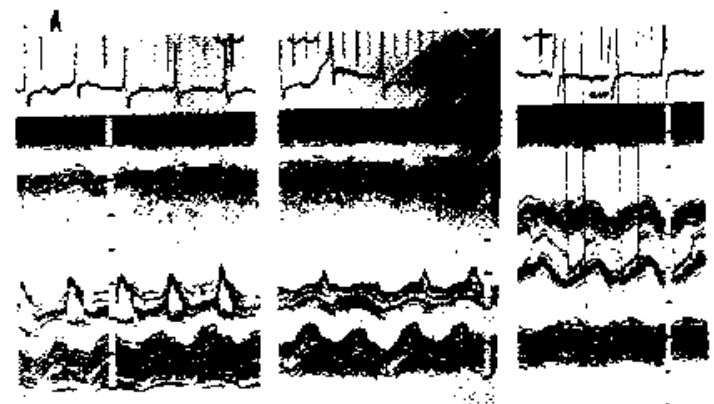

reducida de las valvas mitrales (bajo débito). Además de estos hechos no específicos. en todos nuestros casos se constató un nítido engrosa. miento del endocardio y del aparato sub-valvular mitral (Fig. 3 y 4 ).
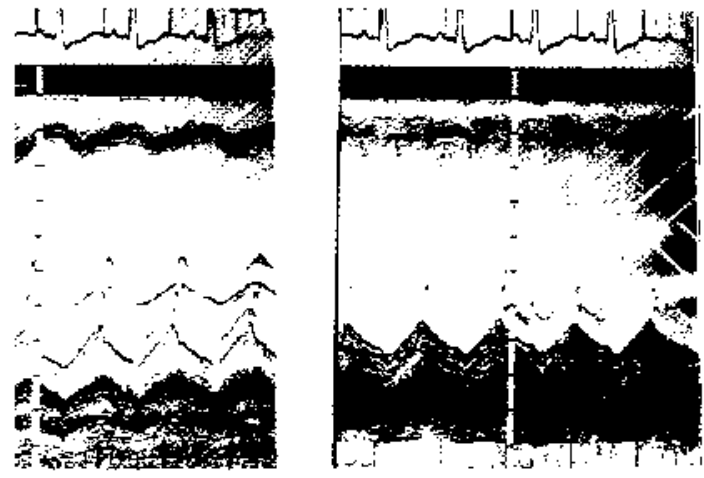

Figura 3: Iribroclastosis endocúrdica. Ecocardiograma Mndo M. Cavidad ventricular izquienda, Marcada dilatacions def ventriculo, septum delgado e hipoquimético, nítido engrosamiento d心 sndocardio.

Ocho pacientes (47\%) fallecieron, 7 antes de ocho meses de evolución y uno, 8 años después del comienzo de sus molestias. Este último enfermo tenía, al fallecer, manifestaciones clínicas de miocardiopatia congestiva, pero en lit necropsia se confirmó el diagnóstico de fibroelastosis. Otro de los niños fallecidos comenzó precozmente (a los 2 meses de edadl con evidencia clínica, electrocardiográfica y ecográfica de miocarditis, evoluciono de manera tórpida: con recaídas y mejorías sucesivas, pero en sus ecocardiogramas seriados de control se observó engrosamiento progresivo del endocardio y del aparato sub-valvular, falleciendo a los 8 meses de edad: su estudio anátomo-patológico demostró una fibroelastosis con clara estratificación del endocurdio hipertrófico en tres capas, en la que, desde la más profunda hacia lì más superficial, se reconocian fibras clásticas y colágenas mís finas y de menor alinidad tintorial (Fig. 5), hallapgo que sugiere que el proceso habia ocurrido en tres

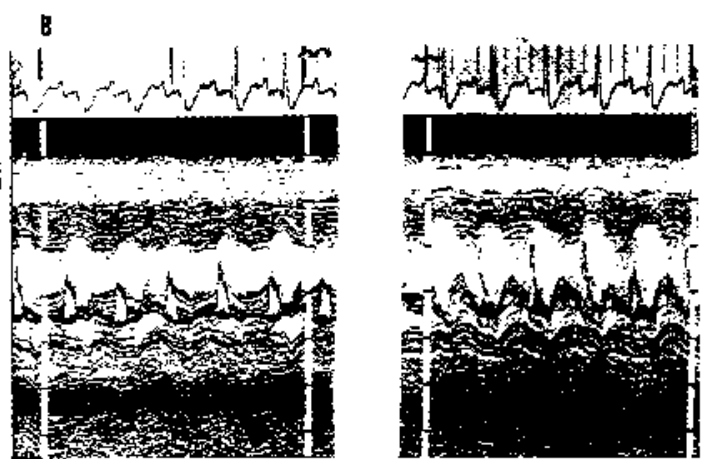

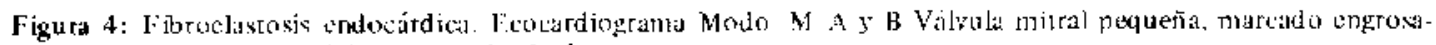
micnto del endocardio y del aparato subvalvulat. 
etapas de distinta antigüedad, correspondiendo lit $_{4}$ capa superficial (interna) al proceso hiperplástico más reciente.

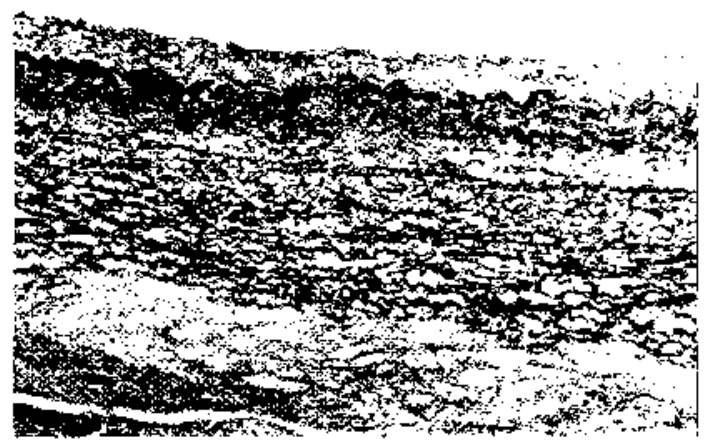

Figura 5: ribroelastosis endocádica del ventriculo kquierdo. Fistratificación de ba hiperplasia fibroclástica del endocasdoo en 3 capas en las que, desde la protiunda a la superficial. disminuyen el grosor $y$ la afinidad tintorial de las fibras coljgenas y elásticas lestas úlimas en negsol. Verhoeff Van Grison. Aumento original 80 $x$ (gentileza Dr. B. Chuatuli, Unjversidad Catolica de (hule).

Nucve pacientes han sobrevivido entre 6 meses y 3.5 anos con un seguimiento promedio de un año. En ellos se ha logrado controlar saltisfactoriamente la insufieiencia cardiaca mediante tratamiento con digitálıcos, djuréticos y vasodilattadores. llamaudo la atención que, a pesar de la manifiesta mejor ía clinica. los parámetros ecoedrdiográficos lo hitcen de modo más leve y lento.

\section{DISCUSION}

Aunque la FEE es una entidad conocida desde hace mucho tiempo, sigue constituyendo un enigma en cuanto a su etiología $2,6-13$. Durarte Jos úlimos anos se ha vuelto en general a la lcoría histórica de la codocarditis fetill y miocarditis. Esta teoria se apoya en la relación de casos de FEE con epidemias de virus Coxsackie B6. el aislamiento de virus ('oxsackie B en ujōos ion FFt. $^{2}$, la producción experimental de FEt en pollos siguiendo a la inducción de curdiopatias por virus de la paroliditis ${ }^{7}$ y la coexistencia de hechos histológicos inflamatorios en pasientes (i) $1 \cdot \mathrm{EE}^{2,8,12}$.

Sólo en cuatro de nuestros pacientes fic posible el estudio virológico, siendo positivo en 3 para virus coxsackie B. Sin embargo, la mayoria de nuestros pacientes $(n=14)$ presentaron síntomals de las vías aéreas superiores y fiebre, probublemente de origen viral. pocos dias antes o concomitantenente con el contenzo de las manifesterciones cardíacas. Esto apoyaria la eliología viral de la ettirmedild.
Sc ha descrito que mientras más precoz es ja presentación de la FEE, más grave es el pronósticol ${ }^{4}$. En nuestros casos, sin embargo, no hubo correlación entre precocidad y gravedad o mortalidad. En cambio hubo correlación entre precocidad e intensidad del tratamiento medico y evolución: $:$ n los últimos 9 casos, en que al tratamiento habitual de digíálicos y diu réticos se agregaron vasodilatidores, la evolución ha sido más benignil, con signos ecocardiográficos de mejoría. Sólo un paciente está aparentemente sano 2.5 años después del comienzo de la enfermedad. La mortalidad promédio en nuestra serje es cercana al $50 \%$ al corto plazo (7 meses promedio de evolución).

Los signos electrocardiográficos de gran reacción ventricular izquicrda diferencian la FEE de la miocarditis ${ }^{15}$. La observación de un nitido engrosamiento del endocardio $y$ del aparato subvalvular mitral en el ecocardiograma Modo-M nos parce otro elenento de indiscutible valor en el diagnóstico de $\mathrm{FEE}$, que no ha sido recalcado explícitamente en la literaturä.

Li demostración histológica, en un paciente fillecido, de compromiso endocárdico en distintas etapas de evolución o antigüedad es de especiátl interés, ya que a nuesro juicio, es la primera descripcion histopatologica de que csta enfermedad puede evolucionar en brotes sucesivos con engrosamiento progresivo fibroclastico del endocardio. Creemos posible que, con tratamiento intenso precor. se podrían preventir los sucesivos brotes que empeorarian el pronóstico y aumentarían la letalidid. De ahi la importancia de hacer oportunamente el diagnóstico con li ayuda de los signos descritos de FEE en el ecocardiograma Modo-M.

\section{RESUMEN}

Se presertan los hallazgos dínicos de 17 pacientes portadores do fibroclastosis endocardiea (FEE). 10 con estudio cocoardiográfico seriado y comprobación necropsica en 8 . $A$ los signos ecocardiográficos de la miocardiopatia congesti$\checkmark a$, en el ecocardiogrami Modo M de estos pacientes se constató un nitido engrosamiento del endocardio $y$ del aparato subvalvular: en un caso, ef engrosamient o progreso claramente hasta el fallecimiento. Los hallazgos histológicos sugicren que la enfermedad puede evolucionar en brotes. con cngrositmiento fibroelástico progresivo del endocardio. La letalidad en cl corto plazo lue cercana a $50 \%$. Pero la evolución depende en gran medicla de la precocidad, intensidad y constanciat del tratamiento. E] uso de vasodilatadores pirce mejorar el pronostico y la sobrevida. 


\section{REFERENCIAS}

1. Folger, G.M. Jr.: Endocardial thoroclcastosis. A continuing and unsolved dilemmia. Clin. Pediat. 10: 246, 1971.

2. Frühling, L: Kurn, R.; Lavillaurtix, J.; Surgus, $A_{\text {.: }}$ Foussereanx, $S$. La myo endocardite chtonique fibro-clastique du nouvcau né et du nourtison (fibro-elastose) Ann, Anat. Pathol. ?: 227, 1962.

3. Sellers, F.J.; Keith, J.D.: Manning, J.A.: D iagnosis of primary cndocardial fibroelastosis. Circulation 29: 49, 1964 .

4. Linte, L.M., Adams, F.: Prognosis in endncardial fibtoclastosis. Am. J. Dis. Child. J 05: 329, 1963.

5. Chung. K.J.: Mamning, J.A.; Gromiak, R.: Presentation - Association of European Pediatric Cardiologists, may 173.

6. Witchell, S.C.: Froehlich, I.A.; Banas, IS.: Gilkerson, M.R.. An epidemiologic askesmunt of primary enducardial tib roclastosis. Am. J. Cardiol. 18: 859, 1966.

7. St Jeme, J.W. Jr,; Peralta, II.; Farias, E.; Dauis, C.W.C.; Noren, G.R.: I.xpetimental gestationa] mumps yirus infertion and condocadial fibroclasto. sis. Pediatrics $48: 821,1971$.
8. Hurchings, G.M.; Vie, S.A.: The progression of interstitial myocanditis to idiopathic cndocardial fibroelastusis. Am. J. Pathol. 66: 483, 1972.

9. Chen, S.: Thompson, M.W., Rose. V.: Endocardial fibroelastosis: Family studies with special reterence to counseling. I. Pcdidtr. 79:385, 19?1.

10. Joffe, H.S.: The role of viruses in heart discase in infants and childzen. Pacdiatr. Cardiol. 4; 699 . 1981.

I1. Keith, J.D.; Rowe, R.D.; Vlad, P.: Hear I Disease in Infanty and Childhood, New York: Mackillan Co. 1978. pp. 941.

12. Schryer, M.J.P.: Karnauchow, P.N.: lindocardial libroclastosis. Etiologic and pathogenetic considerations in child ren. Am. Heart J. 88: 55?, 1974

13 Somerville, $J_{\text {.: }}$ Primary myocardial disease in children ar the myth of fibroelastosis. Procecdings of the A ssoc. Europe. Paed. Cardiologists. I I th Annual General Mceling, may, 1973.

14. Manning, I. F; Sellers, F.J.:Bynum, R. Ketth, J.D. Medical management of clinical endocardial fibroelastosis. Circulation 29: 60, 1964.

15. Vlad, P. Row'e, R.O.: Keith, J.D.: The electrocardiogram in primaty endocardial fibroctastosis. Br Heart J. 17: 189, 1955.

16. Chuoqui, B.: Comuničación personal. 